\title{
Science et rhétorique : la double articulation rhétorique du discours scientifique
}

Jacky Martin

\section{CpenEdition}

\section{Journals}

Édition électronique

URL : http://journals.openedition.org/asp/2661

DOI : 10.4000/asp.2661

ISSN : 2108-6354

\section{Éditeur}

Groupe d'étude et de recherche en anglais de spécialité

\section{Édition imprimée}

Date de publication : 1 décembre 1998

Pagination : 5-16

ISSN : 1246-8185

\section{Référence électronique}

Jacky Martin, «Science et rhétorique : la double articulation rhétorique du discours scientifique », ASp [En ligne], 19-22 | 1998, mis en ligne le 30 janvier 2011, consulté le 30 avril 2019. URL : http:// journals.openedition.org/asp/2661 ; DOI : 10.4000/asp.2661

Ce document a été généré automatiquement le 30 avril 2019

Tous droits réservés 


\title{
Science et rhétorique : la double articulation rhétorique du discours scientifique
}

\author{
Jacky Martin
}

1 Le titre de cette communication est volontairement trompeur: les deux notions qu'il comporte doivent être interprétées dans une relation de complémentarité et non d'opposition. Le statut rhétorique de la science n'est plus à démontrer (Gusfield 1976; Latour \& Fabbri 1977; Mulkay 1979). Il constitue l'une des hypothèses de base pour un grand nombre de chercheurs dans le domaine du fait scientifique: épistémologues, sociologues, linguistes, etc. J'ai souhaité laisser flotter l'ambiguïté afin de souligner le caractère idéologique de l'opposition entre la rhétorique traitant de l'art de la persuasion fondé sur les croyances, et la science correspondant au discours de réalité fondé sur la logique et l'observation. Il semble maintenant bien établi que cette dualité largement partagée par le sens commun ne fait que refléter :

- les différences de statut ou d'investissements d'une société donnée par rapport à la hiérarchie des modes discursifs présents en son sein : ainsi, il est clair que le discours de la science est survalorisé par rapport à nos parlures quotidiennes.

- plus profondément, on a affaire à une occultation (variante des « illusions » mises en évidence par Fuchs et Pêcheux) du rôle du langage dans la communication humaine ; certains types de discours seraient perçus comme transparents, rationnels et consensuels ; d'autres, au contraire, seraient irrémédiablement opaques, artificieux et éristiques et donc, forcément rhétoriques. Alors que, selon beaucoup de chercheurs, il ne s'agit que de modalités de discours dont le statut est fondamentalement rhétorique.

Il est peut-être temps de redéfinir notre conception de la rhétorique. Nous appelons rhétorique toute stratégie de discours orientée vers une certaine finalité : tautologie ou illusion induite par l'effet miroir (ou effet "Vache qui rit») inhérent au langage? Lorsqu'on fait ainsi référence à la rhétorique, on ne fait peut-être, en définitive, que décrire la nature profonde du langage - ce que le langage « dit être » - en d'autres termes, une construction verbale à finalité référentielle. 
3 Il est permis de penser, dans ces conditions, qu'il existe plusieurs façons, plus ou moins conventionnelles, plus ou moins contraintes, d'utiliser l'appareil rhétorique du langage. Face à une utilisation que l'on peut qualifier d'informelle, libre et adaptative dans l'usage commun de la langue, il est possible d'envisager notre premier degré de définition du discours scientifique comme une rhétorique de la contrainte. Il y aurait deux types de fonctions rhétoriques: une rhétorique ouverte et une rhétorique contrainte qui marquent la différence entre deux énoncés pourtant perçus comme assez voisins : (1) «Mon cœur est triste car il pleut sur la ville. » et (2) «Il pleure dans mon cœur comme il pleut sur la ville. » Aucune différence notable du point de vue linguistique sinon qu'en 1 tout accrochage à la situation reste possible alors qu'en 2, à cause du parallélisme établi autour de " comme ", les deux énoncés ont été affectés d'une finalité signifiante en tant que texte. L'usage interactionnel de la langue se caractérise par la multivalence des formes et des fonctions, la polysémie des valeurs et l'indécidabilité des valeurs référentielles; il ne s'agit pas là d'un usage dévalué, vulgaire ou fautif, mais de la condition indispensable pour que s'accomplisse l'interlocution au sens où l'entend Francis Jacques (1985): les participants à l'acte d'interlocution doivent pouvoir à tous moments ajuster et régler leurs énonciations s'ils veulent espérer négocier leurs différences et produire du sens. Le flou, le vague et l'ambiguïté sont constitutifs de la langue ; un langage unique et commun à tous signifierait la fin de toute différence, donc de toute interaction.

4 C'est précisément sur ce projet - négativement défini ci-dessus - que s'échafaude le discours scientifique; non que la communauté scientifique cesse d'interagir - nous savons au contraire qu'elle est agitée de soubresauts et de convulsions -, mais elle décide par convention de définir et tenir pour acquis au niveau cognitif un certain tissu de discours comme représentation d'un certain segment du réel référentiel : on n'est pas très loin, de ce point de vue et paradoxalement, de Verlaine et du discours poétique. Cette fixation ( $\mathrm{d}$ 'où notre " rhétorique de la contrainte ») va complètement à contre courant de la nature profonde, perçue comme "naturelle», du langage. Elle est un prix à payer, comme nous le verrons plus tard, pour la définition d'un autre type de projet.

Cette contrainte en langue se traduit par deux types de prolifération linguistique - que l'on pourrait assimiler à la rhétorique entendue dans le sens commun linguistique.

D'une part, il y a la prolifération directement liée à la contrainte, qui a été assez bien documentée. Dès l'instant que le langage est soumis à trois facteurs de contrainte: la monosémie, la monoréférentialité et la logification ${ }^{1}$, il perd l'élasticité nécessaire pour réaliser des désignations inévitablement variables et imprévisibles. Le discours scientifique se place dans une situation de prévisibilité maximale, d'où, en raison de l'imprévisibilité des observations sur le réel, le bourgeonnement incessant du langage sur lui-même, tant au niveau du lexique que des relations syntaxiques. Ainsi dans le domaine d'étude qui est aujourd'hui le mien, l'immunologie, on est passé des très innocents "globules blancs » aux «lymphocytes », des «lymphocytes » aux «lymphocytes T et B » et, de ces cellules, à diverses références métonymiques par le biais de leurs récepteurs spécifiques qui, à leur tour, recatégorisent ces cellules en " lymphocytes CD4 auxiliaires » et « lymphocytes CD8 cytotoxiques ». La prolifération terminologique ne s'arrête pas là. Si cette explosion lexicale est bien documentée, il n'en va pas de même de phénomènes semblables sur le plan syntaxique. Beaucoup trop d'importance a été accordée à, par exemple, la fréquence du passif dans le discours scientifique alors que nous avons à faire à un redéploiement total des fonctions syntaxiques placées sous la contrainte de la 
causalité. Dans cette optique, on s'aperçoit que les notions (issues de la grammaire interactionnelle) d'agent et de thème disparaissent : « an antigen binds (to) a $B$ cell just like $a$ $B$ cell binds an antigen». Nous avons plutôt affaire à une orientation majeure dans la causalité, qui s'oppose à l'orientation secondaire («when bound/binding»), et à l'explicitation de la causalité : "causes (induces, triggers, effects) to bind». Ces trois modalités du rapport de contiguïté entre deux notions permettent de construire dans la linéarité du discours des cascades de prédicats d'une grande complexité logique.

D'autre part et corrélativement, cette logique de la contrainte est à l'origine d'une logique inverse du détour, du faux-fuyant ou de la périphrase. Alors que la première dynamique vise à éliminer le flou, l'ambigu et la variation, celle-ci consiste à ménager dans un discours, par ailleurs rigoureusement logique, une place pour l'incertain, l'insuffisamment établi ou la nuance. Il s'agit du second volet de la rhétorique de la contrainte qui consiste à réintroduire du libre dans la contrainte : ainsi, au raffinement terminologique que nous avons observé s'opposent des formulations vagues telles que " intracellular infection » ou "antigenic agent » qui, à elles seules, nécessiteraient des pages d'explicitation mais sont laissées dans un état de latence "pour les besoins de la démonstration "; de même, dans l'exemple syntaxique cité plus haut, le recours à la forme nominalisée - qui n'est pas une notion au sens strict de ce terme - semble un défi à la consigne de causalité. Nous posons que ces deux volets de la rhétorique de la contrainte sont les deux aspects complémentaires d'un même projet : il ne peut y avoir de précision terminologique sans une égale part d'imprécision. Cette hypothèse reste à vérifier.

D'où le schéma en figure 1 :

Figure 1

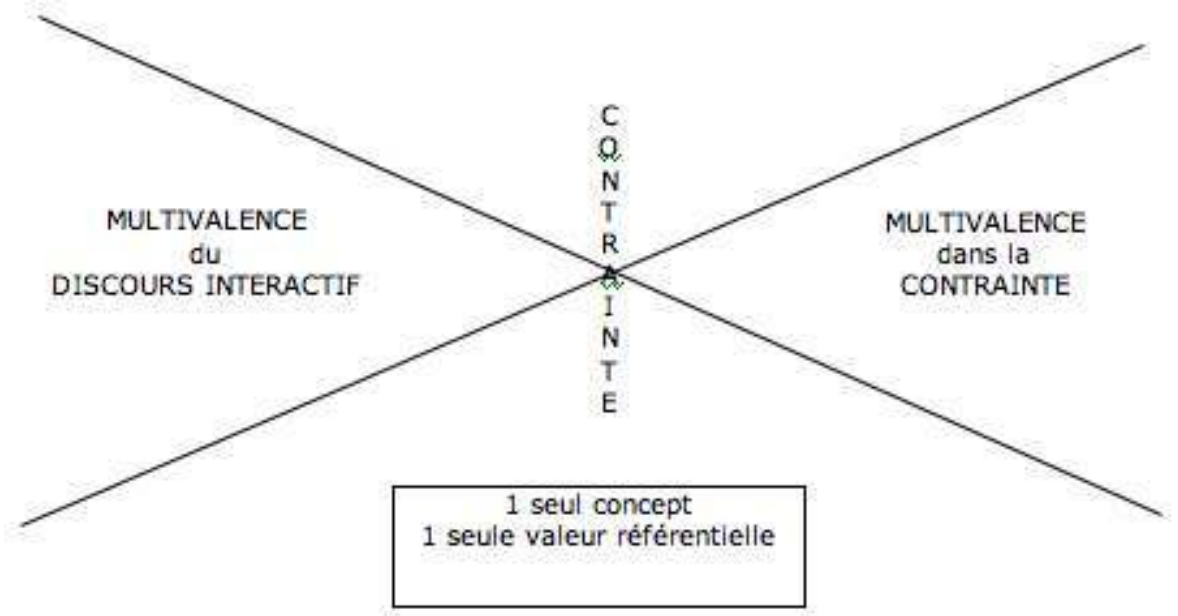

Il semble que le discours de la science, contrairement aux idées reçues, alterne constamment entre les deux dynamiques qui constituent ce discours comme une rhétorique de la contrainte.

9 Nous avons remis en cause et - espérons-le - refondé le concept de rhétorique, il est temps à présent de s'attaquer à celui de "discours scientifique", tout aussi idéologiquement investi et, partant, tout aussi « évident » sur le plan des représentations communes. Même chez ceux qui font profession de s'intéresser au phénomène plus qu'à son contenu conceptuel: épistémologues, sociologues, didacticiens, on ne sait jamais clairement de quoi on parle et sur quoi on raisonne. Si l'on s'accorde généralement sur la science dans son principe, il est plus difficile d'obtenir un accord sur ces manifestations; 
ou plutôt, cela ne semble jamais revêtir une importance cruciale pour la plupart des chercheurs. Certains ont à l'esprit la science en soi comme système cognitif, d'autres font référence à certains de ses domaines, ou bien embrassent dans le même discours tous ces domaines comme s'il n'existait entre eux aucune différence notable; d'autres encore distinguent la « scientificité » en fonction des domaines d'application. De toute évidence, la spécification, que nous avons évoquée plus haut, ne suffit pas pour rendre compte de la diversité, de la stratification complexe et des finalités multiples du discours scientifique.

Comment a-t-on, jusqu'ici, rendu compte de cette diversité ? Deux approches se signalent à l'attention parmi une foule d'études moins caractérisées dans leurs orientations théoriques :

11 1. L'approche néo-rhétoricienne (Swales, Trimble, Sager), centrée sur le concept de genre, se fonde implicitement sur le paradigme mertonien qui représente le discours scientifique comme le « véhicule » d'un savoir fondé sur la logique. Ce savoir vise, par un processus d'émulation entre les différents opérateurs associés à sa construction, à dégager une représentation du monde approchant progressivement de la vérité. Dans cette perspective, le discours a une double fonction: traduire au plus juste un contenu cognitif dont il n'est que le truchement; et l'adapter à son contexte de communication de façon à ce que sa compréhension soit à la fois optimale et appropriée à son contexte de réception.

cept de " genre » est censé synthétiser cette double fonction. Or, il apparait, au vu des récents développements apportés par les sociologues de la science et des thèses "socio-constructivistes" en particulier, que nous avons adaptés pour définir notre propos, qu'en matière de discours scientifique, nous n'avons pas seulement affaire à une composante rhétorique affectant la périphérie d'un hypothétique «noyau » cognitif mais à une rhétorique généralisée affectant le contenu même du discours : ses modèles et ses paradigmes.

13 2. selon les socio-constructivistes, d'horizons aussi divers que les écrits de Mulkay, Latour, ou Myers pour ne citer que ceux-là, le discours scientifique est le produit d'une construction cognitive négociée à partir de l'observable et ratifiée par la communauté scientifique pour rendre compte du réel référentiel. Le texte, dans ces conditions, devient la trace lointaine, souvent méconnaissable, en tout cas difficilement interprétable en tant que telle, d'un processus de «bricolage » expérimental, d'un ajustement progressif des hypothèses et, finalement, d'une rhétorique de persuasion à l'adresse de la communauté scientifique - trace et, en aucun cas, lieu de définition du sens. On comprend, dans ces conditions, pourquoi les sociologues de la science ont manifesté une certaine méfiance à l'égard du texte pris en dehors de son contexte de production.

Pourtant, deux études de Latour (Latour \& Fabbri 1977 ; Latour 1980), une de Gilbert \& Mulkay (Knorr 1980) et celle pionnière de Gusfield (1976) ont jeté les bases d'une nouvelle approche du discours en tant que trace ou vestige de déterminants sociaux ou, dans de récents développements (Latour 1980; Callon 1986), comme élément actanciel dans un réseau d'interactions. Ces études, parfaitement justifiées comme prolongements d'une approche sociologique, ont constitué la base de notre réflexion sur les fonctionnements du texte scientifique. Elles nous paraissent néanmoins insuffisantes d'un point de vue linguistique, dans la mesure où elles mettent en lumière les lignes de fracture du texte plus que ses lignes de construction. Elles «traversent» le texte plus qu'elles ne l'expliquent. 
st temps, de notre point de vue, de requalifier le texte comme construit et non simplement comme résultat d'un processus à définition externe. Si l'on adopte la notion de «réseau» décrite par Callon, il apparaît que le texte, loin d'être une simple composante dans un réseau de déterminants, joue le rôle crucial de foyer d'interférences dans la mesure où tous les autres "actants" du réseau scientifique, quel que soit leur poids par ailleurs (on pense à l'importance des financements de la recherche, par exemple), se trouvent replacés sur un pied d'égalité. Ils sont simultanément déréalisés et retraduits dans le même milieu de discours. Notre étude se situe à cette interface problématique entre le système linguistique et ses déterminants socio-économiques et culturels.

Pour concilier les deux, nous proposons une théorie du discours cognitif fondée sur un double concept, ce qui revient à dire que la spécification scientifique, dont on a vu qu'elle constituait le premier niveau de rhétoricité, assigne au discours scientifique une double orientation : une orientation cognitive visant à reconstruire le réel sous la forme d'un discours cohérent, logique et vérifiable, et une orientation valorisante asservissant cette construction à une valeur et, donc, transitivement, à une finalité.

Les deux orientations sont à la fois complémentaires et nécessaires pour définir le concept de science, comme le schéma figure 2 essaie de le faire comprendre.

Figure 2

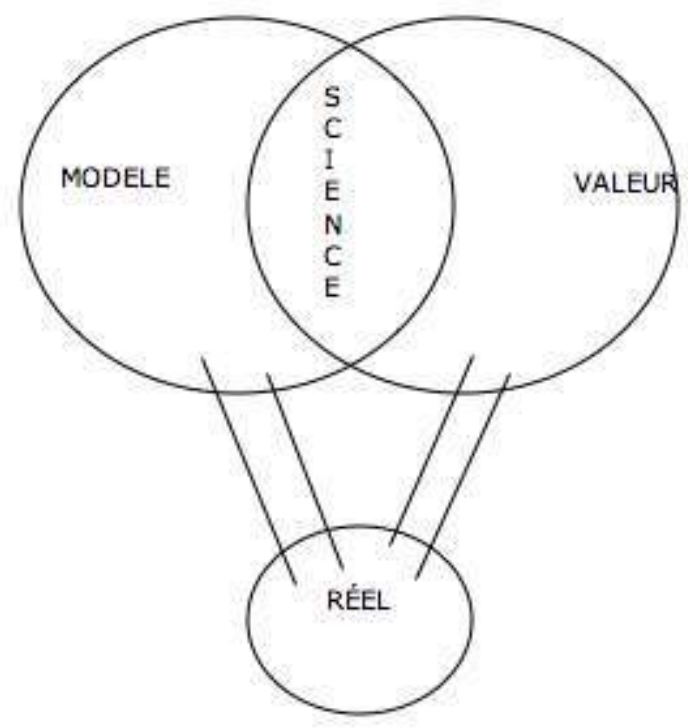

Le modèle se définit comme une remise en ordre du réel ; mais cette construction ne saurait perdurer sans être orientée vers un projet qui simultanément le construit et le déconstruit. Sans valeur d'orientation, le modèle deviendrait rigide et réducteur comme, par exemple, la persistance de la cosmologie ptolémaïque bien après son invalidation; il s'agit, de façon beaucoup plus commune, de ce que Latour appelle la science "froide", celle que l'on définit comme située dans une hypothétique « tour d'ivoire. » Par ailleurs, la valeur à elle seule ne peut se réaliser sans un modèle fondé sur le réel; sans construction cognitive vérifiable, l'orientation vers la valeur dériverait vers l'irréalité et s'apparenterait à quelque chose comme de la « science-fiction. » Nous avons affaire, non pas à des rapports linéaires, mais à des cercles d'interactions. 
19 Le projet cognitif se réalise dans ce que nous appelons le Macrotexte qui ne saurait être assimilé, ni à un simple lexique de spécialité (insuffisant en lui-même pour définir la scientificité) ni au savoir des spécialistes (la faculté d'assigner des référents précis à des textes). Il s'agit d'un tissu relativement stable de notions et de relations prenant en charge le lexique et sans l'aide duquel le savoir des spécialistes ne pourrait s'exprimer un pré-construit discursif, en quelque sorte, partagé par une communauté scientifique donnée.

20 Mais, ce Macrotexte n'est jamais totalement réalisé en tant que tel, sauf dans des textes qui ont perdu toute actualité et donc, toute force discursive. Lorsqu'ils sont porteurs d'un enjeu, ils donnent lieu à des Stratégies de production qui visent à inscrire le Macrotexte en discours. Le Macrotexte est littéralement réécrit et reconverti dans l'axe de valeurs qu'il faut bien qualifier d'idéologique. Nous n'avons plus affaire, comme dans les approches rhétoriciennes, à une mise en forme périphérique du discours. C'est l'ensemble du macrotexte qui se trouve redéfini en fonction de déterminants socio-économiques.

21 Si l'on peut comparer le lexique de spécialité aux différents types et valeurs de cartes et de figures qui composent un jeu de cartes, le Macrotexte s'apparente plutôt aux différents jeux que l'on peut pratiquer à partir d'elles; et les stratégies aux parties effectivement jouées, c'est à dire, pour poursuivre notre métaphore, les textes effectivement produits. Cette combinaison de la stabilité (Macrotexte) et des variations (Stratégies) forme la deuxième articulation rhétorique dudiscours scientifique.

Les trois stratégies fondamentales, et donc les trois valeurs assignées à la définition du Macrotexte, peuvent être caractérisées : il y a les stratégies de véridiction (rhétorique de la probation), les stratégies de la communication (rhétorique de l'information et de la diffusion) et les stratégies de l'appropriation (rhétorique de la récupération [idéo]logique).

Nous avons choisi d'illustrer certaines procédures liées à la rhétorique de la véridiction qui correspond au discours scientifique tel qu'il est communément entendu. Nous appuierons notre propos sur quelques observations portant sur un compte rendu de recherche publié dans la revue Science dans le domaine de l'immunologie ${ }^{2}$.

Figure 3 


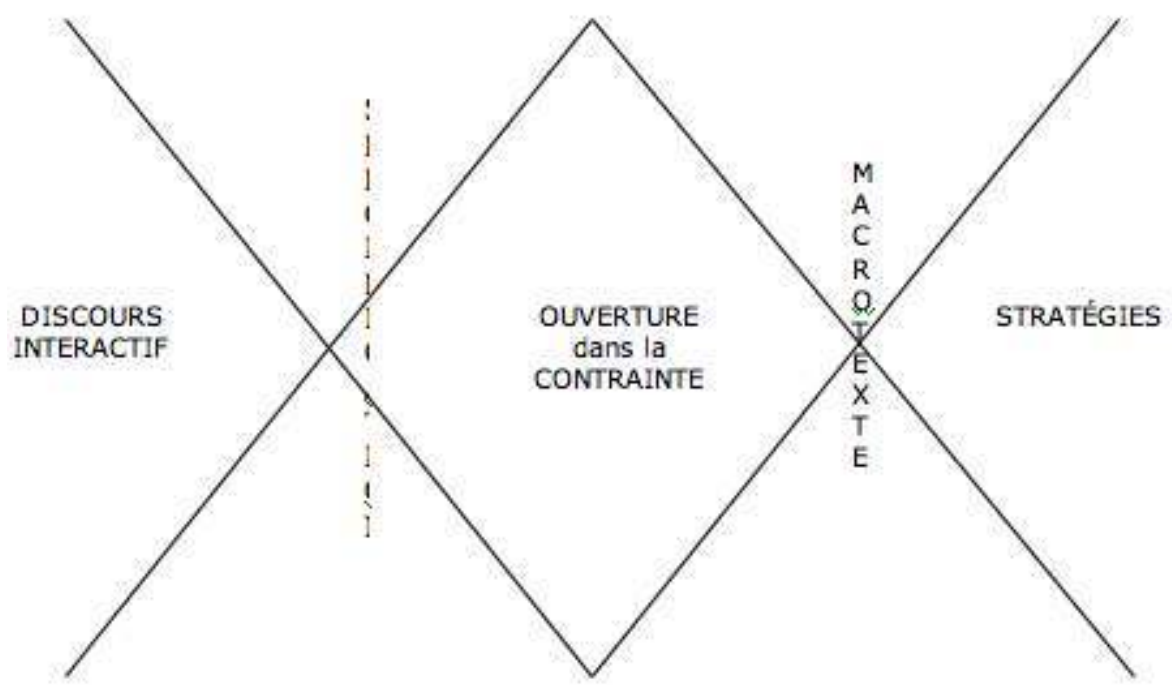

\section{LA DOUBLE ARTICULATION DU DISCOURS SCIENTIFIQUE}

24

et à le retrancher du flot des énonciations communes: il s'agit, en d'autres termes, d'établir son "autorité » en tant que texte produit par un groupe de savants pour une communauté de spécialistes, à partir d'une sélection sévère quant à la recevabilité de son contenu, sélection effectuée par les experts et les arbitres de la revue Science. Il s'agit, par ailleurs d'installer ce texte dans un univers terminologique restreint, celui que nous avons défini comme la Macrotexte.

Si le premier niveau concerne l'accréditation du texte, le second niveau concerne sa validation. Elle s'effectue de deux façons différentes qui, toutes les deux, la chose est notable, font référence à des textes. Le premier type de textes est ce que l'on pourrait appeler la «doxologie» du discours immunologique ou l'ensemble de tout ce qui est dicible en ce domaine. Cela concerne tous les passages dans le texte qui sont référencés à des entrées citées dans la bibliographie. La communication scientifique s'inscrit dans la continuité (Le Macrotexte est condamné à s'élargir sauf à devenir une science " froide ») 
et dans le défaut (ce texte est sans cesse menacé dans sa crédibilité) du discours accrédité par la communauté des spécialistes. Ce point semble assez bien admis par l'ensemble de ceux qui étudient le texte scientifique : le Texte Scientifique est à la fois éristique et doxologique; c'est sur cet aspect que portent pour l'essentiel les études d'inspiration néo-rhétoricienne.

Moins largement acceptée est la nature textuelle de la deuxième forme d'accréditation, la référence aux comptes rendus d'expériences qui, pour beaucoup encore, sont considérés comme la traduction fidèle et immédiate du réel. Or, comme l'a montré Latour dans Laboratory Life, nous avons affaire à des interprétations d'«inscriptions» (traces produites par les différents appareils de mesures ou d'enregistrement), donc à des textes, produits par des machines, elles-mêmes assimilables à ce que Bachelard désignait déjà en 1953 comme de la «théorie (et donc, encore une fois, du texte) réifiée." Le discours scientifique trace donc la cohérence entre ces différents textes qui, sans lui, demeureraient épars et donc non signifiants.

Le troisième niveau de ce texte est proprement stratégique. Il consiste à prouver l'existence d'un objet appelé « chaine $B$ du récepteur d'antigène des leucocytes $T$ ». Trois procédures complémentaires vont être mises en œuvre : la réification, c'est-à-dire tout ce qui tendra à prouver qu'un tel objet existe effectivement, d'où le recours aux tableaux et aux figures qui placent le discours en retrait et au service d'une réalité qui le domine. Or, il est assez simple de voir, sans être très expert en immunologie, que cette image du réel n'a pu être obtenue qu'en dissociant les deux parties du TCR, les chaînes A et B, et qu'une partie importante de l'article est consacrée à combattre la possibilité d'un très probable artefact induit par cette dissociation. Par ailleurs, reste non explicitée et donc considérée comme acquise, l'analogie sur laquelle repose la totalité de l'article, à savoir que le TCR des lymphocytes $\mathrm{T}$ serait formé sur le modèle des immunoglobulines sécrétées par les leucocytes B. Or, même s'il s'agit d'une hypothèse plausible, il n'est question à ce point de la recherche que d'une pure et simple supputation. On voit bien comment l'objet de ce texte se situe dans le présupposé d'une stabilité terminologique et dans l'extension d'un certain savoir paradigmatique (la structure des immunoglobulines), dans le cadre d'un raisonnement par analogie: les trois niveaux de la stratégie de véridiction ont ainsi fusionnés.

La seconde procédure de probation est mieux connue et concerne le processus d' externalisation. Il s'agit de montrer que cet « objet de discours » existe indépendamment de l'observateur qui est dès lors un témoin privilégié mais absent : d'où le jeu complexe des passivations et des pronoms personnels, ainsi que les modalisations graduées sur lequel je ne m'attarderai pas. Le troisième type d'opérations de véridiction, encore mal étudié, concerne la logification de ce discours, c'est-à-dire faire en sorte que ce qui est apparu de façon fortuite ou désordonnée dans le cadre du laboratoire, soit perçu comme une démonstration sans faille par laquelle tout converge vers une conclusion indiscutable. Je me contenterai d'attirer l'attention sur un seul point de ce texte, commun à tous les autres articles de recherche : la conclusion est posée d'avance, ce qui suppose : (1) que le texte n'est qu'une circonlocution, nécessaire mais accessoire, pour nous ramener à ce résultat posé d'avance; (2) qu'il n'y a pas d'autres possibilités d'interprétation des faits en question et, plus important, (3) qu'un contenu informationnel a été produit, un noyau dur de vérité, détachable des conditions de sa production. 
31 C'est sur ces trois ordres d'illusion (ou de «valeurs véridictives » dans mon jargon) que repose la "vérité du discours » scientifique. Nous pensons avoir suffisamment montré, que sans vouloir dénigrer le projet de la science, les contenus cognitifs qu'elle propose ne sont en fin de compte que de constructions textuelles qui ressortissent donc à une analyse discursive.

En conclusion, tout en regrettant le caractère exagérément allusif de notre exposé, tant sur le plan théorique que sur celui de l'exemplification, notre étude permet de montrer, ou de proposer à la discussion, que :

- contrairement à la pratique de sociologues de la science qui mettent en doute l'accessibilité du texte scientifique sans référence à son contexte d'engendrement (Myers 1990), il faut requalifier le texte scientifique et l'étudier dans sa variété typologique, sa structure interne et sa dissémination. Si toutes les marques textuelles ne sont pas toujours lisibles sans une bonne connaissance de la spécialité en question, c'est au niveau textuel et à ce niveau seul qu'elles se réalisent et qu'elles sont interprétées et accréditées par la communauté scientifique ;

- qu'il est urgent de combiner les acquis de recherche des néo-rhétoriciens à ceux des sociologues de la science;

- que pour mener à bien cette tâche, un nouveau corps d'hypothèses théoriques est nécessaire réconciliant les deux aspects fondamentaux du discours scientifique : son orientation vers la variation dans la spécification.

Nous pensons y être parvenu, en posant les notions corrélées de Macrotexte et de Stratégies, qui sont de nature à rendre compte du statut hybride de notre objet, à michemin entre sociologie et linguistique.

\section{BIBLIOGRAPHIE}

Callon, Michel. 1986. « Some elements of sociology of translation: Domestication of the scallops and the fishermen of St Brieuc Bay ». In Law, John (dir.), Power, Action and Belief: A New Sociology of Knowledge. Londres : Routledge, 196-223.

Fuchs, Catherine et Michel Pêcheux. 1985. « Mises au point et perspectives à propos de l'analyse automatique du discours ». Langages 37, 7-80.

Gilbert, Nigel \& Michael Mulkay. 1980. «Contexts of scientific discourse: Social accounting in experimental papers ». In Knorr, Karin \& R. D. Whitley (dir.), The Social Process of Scientific Investigation, Sociology of the Sciences Yearbook, Vol. 4. Dordrecht and Boston, MA : Reidel, 269-294. Gusfield, Joseph. 1976. « The literary rhetoric of science : Comedy and pathos in drinking driver research ». American Sociological Review 41, 16-33.

Jacques, Francis. 1985. L'Espace logique de l'interlocution. Paris : Presses universitaires de France.

Knorr, Karin \& R. D. Whitley (dir.). 1980. The Social Process of Scientific Investigation, Sociology of the Sciences Yearbook, Vol. 4. Dordrecht and Boston, MA : Reidel. 
Knorr-Cetina, Karin \& Michael Mulkay (dir.). 1983. Science Observed. Perspectives on the Social Study of Science. Londres : Sage.

Latour, Bruno. 1980. « Is it possible to reconstruct the research process? ». In Knorr, Karin \& R. D. Whitley (dir.), The Social Process of Scientific Investigation, Sociology of the Sciences Yearbook, Vol. 4. Dordrecht and Boston, MA : Reidel, 53-75.

Latour, Bruno et P. Fabbri. 1977. « Pouvoir et devoir dans un article de science exacte ». Actes de la Recherche en Sciences Sociales 13, 81-95.

Latour, Bruno \& Steve Woolgar. 1979 [1986]. Laboratory Life. The Construction of Scientific Facts. Princeton, NJ : Princeton University Press, Sage.

Martin, Jacky. 1996. «Les enjeux du discours scientifique : la stratégie de vérédiction ». ASp 11-14, $13-31$.

Mulkay, Michael. 1979. Science and the Sociology of Knowledge. Londres : Allen \& Unwin.

Myers, Greg. 1990. Writing Biology. Texts in the Social Construction of Scientific Knowledge. Madison, WI : University of Wisconsin Press.

Sager, Juan C., David Dunkworth \& Peter F. McDonald. 1990. English Special Languages. Principles and Practice in Science and Technology. Wiesbaden : Oscar Brandsletter Verlag KG.

Swales, John. 1990. Genre Analysis. English in Academic and Research Setting. Cambridge : Cambridge University Press.

Trimble, Louis. 1985. English For Science and Technology. Cambridge : Cambridge University Press.

\section{NOTES}

1. Gusfield a montré la corrélation qui existe entre ces trois facteurs. C'est précisément parce que des concepts sont posés comme monosémiques et monoréférentiels qu'ils deviennent envisageables en termes purement logiques.

2. G.A Bentley, G. Boulot, K. Karjalainen \& R. Marriuzza. 1995. «Crystal Structure of the b chain of a T Cell Antigen Receptor ». Science 267,1984-87.

\section{RÉSUMÉS}

À partir d'une comparaison entre les notions traditionnelles de rhétorique et de science, une nouvelle définition du discours scientifique est proposée fondée sur un double principe de structuration: la spécification du lexique et de la morpho-syntaxe et l'inscription d'un macrotexte paradigmatique orienté par des stratégies rhétoriques.

Starting from a parallel between the traditional conceptions associated with Rhetoric and Science, a new definition of scientific discourse is put forward, founded on double-sided structuration: the specification of lexicon and morpho-syntactic elements and a combined inscription of a paradigmatic macrotext and rhetorical strategies. 
INDEX

Mots-clés : discours scientifique, macrotexte, rhétorique, spécification, stratégie

Keywords : macrotext, rhetoric, scientific discourse, specification, strategy

\section{AUTEUR}

\section{JACKY MARTIN}

Jacky Martin est professeur des universités à l'université Paul-Valéry Montpellier 3. Sa recherche est axée essentiellement sur les problèmes d'analyse du discours scientifique et technique et sur la traductologie. jacky.r.martin@neuf.fr 